\title{
Designing Pattern-Recognition Surfaces for Selective Adsorption of Copolymer Sequences Using Lattice Monte Carlo Simulation
}

\author{
Arthi Jayaraman, Carol K. Hall, ${ }^{*}$ and Jan Genzer \\ Department of Chemical Engineering, North Carolina State University, Raleigh, North Carolina, USA
}

(Received 19 November 2004; published 24 February 2005)

\begin{abstract}
We describe a simulation method to design surfaces for recognizing specific monomer sequences in copolymers. We fix the monomer sequence statistics of the $A B$ copolymers on a surface containing two types of sites and allow the simulation to iterate towards an optimal surface pattern that can recognize and selectively adsorb the sequence in the copolymer. During the simulation the surface pattern is designed by switching identities of two randomly picked sites. For copolymers with less blocky sequences the designed surfaces recognize the correct sequence well when the segment-surface interactions dominate over the intersegment interactions. For copolymers with more blocky sequences recognition is good when the segment-surface interactions are only slightly stronger than the intersegment interactions.
\end{abstract}

DOI: 10.1103/PhysRevLett.94.078103

PACS numbers: 87.15.Aa, 87.68.+z

Adsorption of polymers on solid surfaces plays an important role in many applications including adhesion [1], chromatography [2], biomedical implant modification [3], etc. In many of these applications it is desirable to have surfaces that "recognize" and selectively adsorb specific monomer sequences along the adsorbing polymers. Recent theoretical studies of the adsorption of random copolymers on heterogeneous surfaces suggest that random copolymers with a given sequence distribution recognize compatible patterns on the surface and adsorb on them [4-7].

The aim of our research is to design optimum surfaces for recognizing specific monomer sequences in copolymers. This work could have practical implications in designing smart sensors that are capable of discriminating between different analytes or toxins, in discovery of new drugs that bind to specific receptors, and in the development of chromatographic materials for target separations. Over the past decade, pattern recognition by polymers has been studied extensively using experiment [8,9], theory [10-12], and computer simulation [4-7,1315]. Muthukumar [13] used dynamic Monte Carlo simulations to explore the recognition of different patterns on surfaces by polyelectrolyte chains. The recognition process was found to take place in two stages: a fast binding of the chain to the surface without any recognition of the surface pattern, followed by a slow relaxation or reorganization process during which the chain slowly adsorbs in a complete pattern-matched configuration on the surface. On the basis of these and subsequent studies [4-7,15], two parameters, namely, the width of the sequence distribution and the width of the surface site distribution, were identified as being the most important variables controlling the adsorption of copolymers on a heterogeneous surface.

Like any thermodynamic process, polymer adsorption on a surface is governed by entropic and enthalpic contributions to the free energy. In his work on the role of entropy in pattern recognition, Muthukumar [14] demonstrated how the formation of chain loops during adsorption influences the chain entropy at different stages of adsorption. Studies on the effect of specific intersegment interactions on pattern recognition were performed by Srebnik [15]. They studied how the magnitude of the intersegment interactions $(|b|)$ affected the surface disorder strength $\left(\sigma_{\tau}\right)$ at which the transition from weak to strong adsorption took place. Srebnik [5] also explored the effects of solvent quality on adsorption of random copolymers on a heterogeneous surface using mean-field theory. Genzer used a 3D self-consistent field model to study the adsorption of copolymers from a copolymer-homopolymer mixture $[10,11]$ onto a physically flat heterogeneous surface composed of two chemically different regions arranged either in checkerboard or random patterns. He demonstrated that copolymers recognize the patterns on the heterogeneous surface and propagate the pattern from the surface to the bulk, thus transcribing them into three dimensions. In recent studies, Golumbfskie et al. [7] investigated this pattern matching phenomenon by studying the kinetics of adsorption of a random copolymer on a randomly patterned surface using dynamic lattice Monte Carlo simulations.

All of the research described above provides valuable insight into pattern recognition. However, the question of how to design optimal surfaces or what pattern the surfaces should have for recognizing specific monomer sequences in copolymers has not been answered yet. In this Letter we describe a method for "training" a surface to recognize a pattern (monomer sequence) in the copolymers.

We perform lattice Monte Carlo (MC) simulation on a cubic lattice in the $N V T$ ensemble. The simulation box of size $24 \times 24 \times 48$ has periodic boundary conditions in the $x$ and $y$ directions, an impenetrable surface at $z=49$, and an adsorbing surface at $z=0$. We consider a system of $A B$ copolymers of length 24 with six possible monomer sequences: $\left[A_{1} B_{1}\right],\left[A_{2} B_{2}\right],\left[A_{3} B_{3}\right],\left[A_{4} B_{4}\right],\left[A_{6} B_{6}\right]$, and $\left[A_{12} B_{12}\right]$ and a surface containing two types of sites: 1 and 2. The segment-surface interactions, $\epsilon_{A 1}$ and $\epsilon_{B 2}$, and intersegment interactions, $\epsilon_{A A}$ and $\epsilon_{B B}$, are considered to 
be attractive. The intersegment interaction potential is short ranged; it only affects nonbonded nearest neighbors. The segment-surface interaction potential is also short ranged, acting only between the sites on the adsorbing surface $(z=0$ plane) and the segments that are on the $z=$ 1 plane. The simulation is split into two steps: design and test. In the design step we "train" the surface to recognize the monomer sequence by adsorbing a bulk of copolymers of the same monomer sequence. In the test step we introduce the designed surface to a mixture containing the six types of copolymers and observe if the surface recognizes and selectively adsorbs the copolymer with the right sequence.

The design step proceeds in the following manner. The system consisting of 60 copolymer chains with monomer sequence $\left[A_{x} B_{x}\right]$ is placed on top of a surface consisting of equal numbers of type 1 and type 2 sites. The initial configuration of the surface comprises randomly placed type 1 and 2 sites. The initial configuration of the chains is obtained by growing each chain as a self-avoiding walk on a cubic lattice. In the initialization stage of the design step we let the system run through $10000 \mathrm{MC}$ steps, moving the chains in the box using standard chain moves: reptation (50\%), kink jump (25\%), and crankshaft moves (25\%), when the segment-segment and segment-surface interactions are turned off and the site switches are not attempted. In the equilibration stage, we let the system run through $10.0 \times 10^{6}$ MC steps while the segment-segment and segment-surface interactions are turned on. The chain segments are subjected to the usual assortment of moves, and sites 1 and 2 are subjected to identity switches. The type of move, chain, and the segment number along the chain are all picked randomly. In one Monte Carlo step (MC step) we make $N_{\text {chains }} \times N_{\text {beads }}$ moves, where $N_{\text {chains }}$ is the number of chains in the simulation box and $N_{\text {beads }}$ is the number of segments in each chain. The probabilities of making a surface site switch or a chain move are equal. The acceptance criteria for the chain moves and the surface site switches are based on the Metropolis algorithm. After equilibrium is attained the final designed surface should have an optimal pattern that recognizes the copolymer sequence that was used in its design, $\left[A_{x} B_{x}\right]$. We repeat this design process to obtain surface patterns for each of the six types of copolymer sequences. In order to quantify the distribution of type 1 and type 2 sites on the designed surface we use an order parameter (OP) defined by

$$
\mathrm{OP}=\left(\sum_{i=1}^{N_{\text {sites }}} \sum_{j=1}^{z} S_{i} S_{j}\right) / z N_{\text {sites }},
$$

where $N_{\text {sites }}$ is the total number of sites on the surface, $i$ and $j$ are nearest neighbor sites, $z(=4)$ is the number of neighbors surrounding each site, and $S$ represents the "spin" for each site $(S=+1$ for type 1 site and $S=-1$ for type 2 site). The order parameter quantifies the extent of patchiness on the designed surface. The value of OP varies from +1 (a completely homogeneous surface with all type
1 sites or all type 2 sites) to -1 (a perfectly alternating surface). We monitor the value of the order parameter during the design process. For each type of copolymer, we take averages of the equilibrium order parameter at the end of ten trials of the design process and compute the standard deviations.

The test step proceeds in the following manner. A mixture of equal numbers (30) of all six types of copolymers is placed on top of the designed surface. The initial configuration of the surface is the same as that of the final surface designed for one type of copolymer in the design simulation. The initial configuration of the chains is obtained in the same manner as in the design process. The initialization stage is similar to that used in the design step. In the equilibration stage, the segment-segment and segmentsurface interactions are turned on and the chain segments are moved. After equilibrium is attained the surface should have selectively adsorbed copolymer chains with sequences that are the same as the chains that were used to design the surface. During the test simulation the surface adsorbs a number of chains of each type of copolymer. In order to quantify the affinity of the surface for a particular copolymer we monitor the selectivity of the surface for each type of copolymer. The selectivity, $S$, for a copolymer chain $\left[A_{x} B_{x}\right]$ is defined as

$$
S_{\left[A_{x} B_{x}\right]}=N_{\left[A_{x} B_{x}\right]} / N_{\text {total }},
$$

where $N_{\left[A_{x} B_{x}\right]}$ is the number of adsorbed $\left[A_{x} B_{x}\right]$ copolymer chains, where $x=1,2,3,4,6$, and 12 , and $N_{\text {total }}$ is the total number of adsorbed chains of all types. A chain is considered adsorbed when at least one of its segments is on $z=1$. A high number of adsorbed chains of a copolymer means that the selectivity for the copolymer is high. For each designed surface we carry out 15 test trials and obtain averages and error bars of the $S_{\left[A_{x} B_{x}\right]}$ calculated at the end of the equilibration stage of the test trial for each $x$.

In order to study the effect of interaction potentials on the selectivity of the designed surfaces, we repeat the design and test with three sets of values for the interaction potentials set I: $\epsilon_{A A}=\epsilon_{B B}=-0.5 \mathrm{kT}$, set II: $\epsilon_{A A}=\epsilon_{B B}=$ $-0.25 \mathrm{kT}$, and set III: $\epsilon_{A A}=\epsilon_{B B}=0 \mathrm{kT}$. In all three sets we keep the strength of the segment-surface interactions constant $\epsilon_{A 1}=\epsilon_{B 2}=-2 \mathrm{kT}$. Therefore from set I to set III the strength of the segment-surface interaction increases relative to that of the intersegment interaction.

Figure 1 shows examples of the designed surfaces obtained for each type of copolymer from one run at set I interactions. The designed surfaces reveal that as the blockiness of the monomer sequence in the copolymer increases, the patchiness increases. Table I lists the average order parameter of the designed surface for each type of copolymer at the three interaction potential sets. For all sets of interaction potentials, as the blockiness of the monomer sequence increases the order parameter increases. For each type of copolymer, as the intersegment 


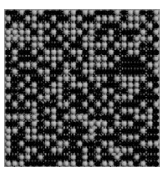

$\left[A_{1} B_{1}\right.$ ]

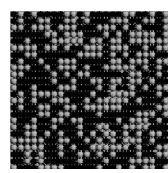

$\left[\mathbf{A}_{2} \mathbf{B}_{2}\right]$

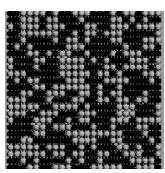

$\left[\begin{array}{l}\mathbf{A}_{3} \mathbf{B}_{3} \\ \mathbf{3}\end{array}\right.$

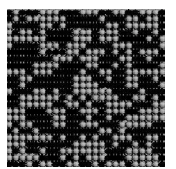

$\left[\mathbf{A}_{4} \mathbf{B}_{4}\right]$

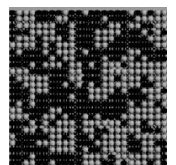

$\left[\mathbf{A}_{6} \mathbf{B}_{6}\right]$

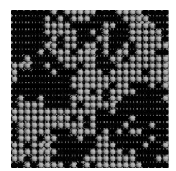

$\left[\mathbf{A}_{12} \mathbf{B}_{12}\right]$

FIG. 1. The surfaces designed for $\left[A_{1} B_{1}\right],\left[A_{2} B_{2}\right],\left[A_{3} B_{3}\right],\left[A_{4} B_{4}\right],\left[A_{6} B_{6}\right]$, and $\left[A_{12} B_{12}\right]$ copolymers with set I interactions.

attraction increases (set III to set I) the order parameter increases.

In Fig. 2 we plot the selectivity (ordinate) for each type of copolymer (abscissa) by the surfaces designed for the various copolymers and interaction potentials. High selectivity for the copolymer which the surface is designed to recognize indicates good pattern recognition. The surface designed for $\left[A_{1} B_{1}\right]$ copolymers [Fig. 2(a)] exhibits good pattern recognition for all interaction potentials, the best recognition being for set III (dotted line). The surfaces designed for $\left[A_{2} B_{2}\right]$ [Fig. 2(b)] and $\left[A_{3} B_{3}\right]$ [Fig. 2(c)] copolymers exhibit good recognition for set III (dotted line) interactions. The surfaces designed for $\left[A_{4} B_{4}\right]$ [Fig. 2(d)] and $\left[A_{6} B_{6}\right]$ [Fig. 2(d)] copolymers have higher selectivity for copolymers with sequences that are blockier than the right sequence. The surface designed for $\left[A_{12} B_{12}\right]$ copolymers [Fig. 2(f)] demonstrates the best recognition for set I (solid line) interactions.

Based on Fig. 2 the best recognition for the copolymers with less blocky sequences $\left(\left[A_{1} B_{1}\right],\left[A_{2} B_{2}\right],\left[A_{3} B_{3}\right]\right)$ is achieved with segment-surface interactions much stronger than the intersegment interactions (set III). When the segment-surface interactions are much stronger than the intersegment interactions, each copolymer adsorbs on the surface without bringing segments of the same type together, as it is energetically more favorable for segments to be in contact with the surface than with each other. This leads to smaller patches of the same type of sites on the designed surface. Smaller patchiness increases selectivity for the copolymers with less blocky sequences. For the copolymers with more blocky sequences, i.e., $\left[A_{12} B_{12}\right]$, the best recognition is achieved with segment-surface interactions just slightly stronger than the intersegment interactions (set I). When the segment-surface interactions are only slightly stronger than the intersegment interactions, each copolymer brings segments of the same type together and adsorbs in a compact folded conformation, as it is energetically favorable for a segment to have segments of

TABLE I. The order parameter (OP) of the designed surfaces for each copolymer type for set I, set II, and set III interactions.

\begin{tabular}{lrrr}
\hline \hline Copolymer & \multicolumn{1}{c}{ Set I } & \multicolumn{1}{c}{ Set II } & Set III \\
\hline$\left[A_{1} B_{1}\right]$ & -0.147 & -0.203 & -0.252 \\
{$\left[A_{2} B_{2}\right]$} & 0.055 & 0.024 & 0.003 \\
{$\left[A_{3} B_{3}\right]$} & 0.098 & 0.065 & 0.044 \\
{$\left[A_{4} B_{4}\right]$} & 0.163 & 0.120 & 0.088 \\
{$\left[A_{6} B_{6}\right]$} & 0.229 & 0.172 & 0.131 \\
{$\left[A_{12} B_{12}\right]$} & 0.345 & 0.257 & 0.192 \\
\hline \hline
\end{tabular}

the same type and the surface adjacent to it. This leads to large patches on the designed surface. Higher patchiness increases selectivity for the copolymers with blocky sequences.

The recognition was poor at all values of the interaction potentials for surfaces designed to adsorb $\left[A_{4} B_{4}\right]$ and $\left[A_{6} B_{6}\right]$ copolymers. This is likely because the surfaces are more patchy (have a larger order parameter) than is required for good pattern recognition. Therefore in order to reduce the patchiness (order parameter), we design surfaces which contain neutral sites, in addition to sites of type 1 and type 2 . The neutral sites (type 0 ) have athermal interactions with segments $A$ and $B$, i.e., $\epsilon_{A 0}=\epsilon_{B 0}=$ $0 \mathrm{kT}$. The presence of neutral sites helps avoid the adsorption of the wrong copolymers by affecting the patchiness on the surface. Depending on the copolymer sequence we want to recognize, there is an optimum percentage of neutral sites that minimizes the adsorption of the wrong copolymers.

A surface with $10 \%$ neutral sites [Fig. 3(a)] designed for the $\left[A_{4} B_{4}\right]$ copolymers has a high selectivity for $\left[A_{4} B_{4}\right]$ copolymer indicating better pattern recognition than the surface with $0 \%$ neutral sites, but only by a small margin. The $30 \%$ and $50 \%$ cases did not show better recognition than the $0 \%$. For $\left[A_{6} B_{6}\right]$ copolymers $30 \%$ neutral sites seem to give the surface an optimal patchiness that improves the recognition of $\left[A_{6} B_{6}\right]$ copolymer [Fig. 3(b)].

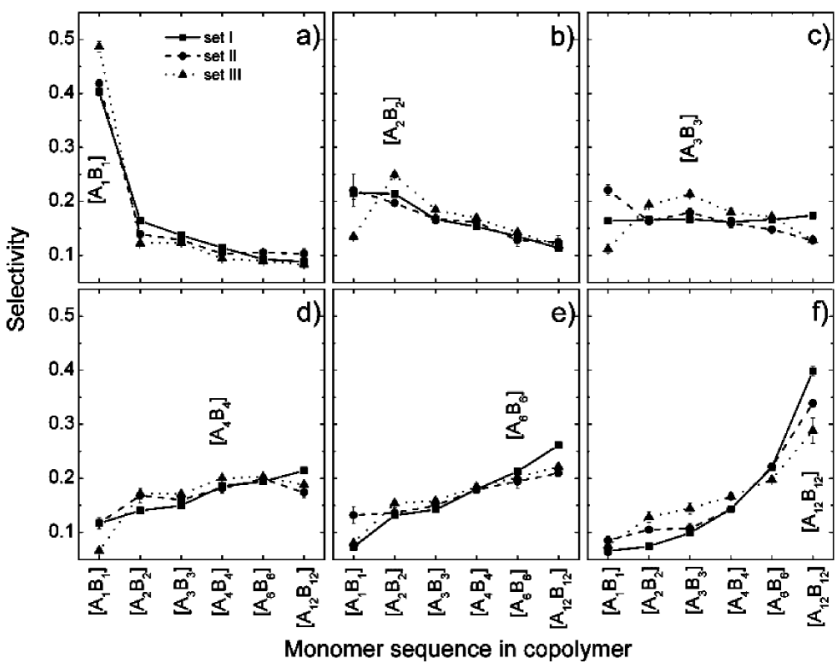

FIG. 2. The selectivity of the surfaces designed for copolymers (a) $\left[A_{1} B_{1}\right]$, (b) $\left[A_{2} B_{2}\right]$, (c) $\left[A_{3} B_{3}\right]$, (d) $\left[A_{4} B_{4}\right]$, (e) $\left[A_{6} B_{6}\right]$, and (f) $\left[A_{12} B_{12}\right]$ with set I (squares + solid line), set II (circles + dashed line), and set III (triangles + dotted line) interaction potentials. 


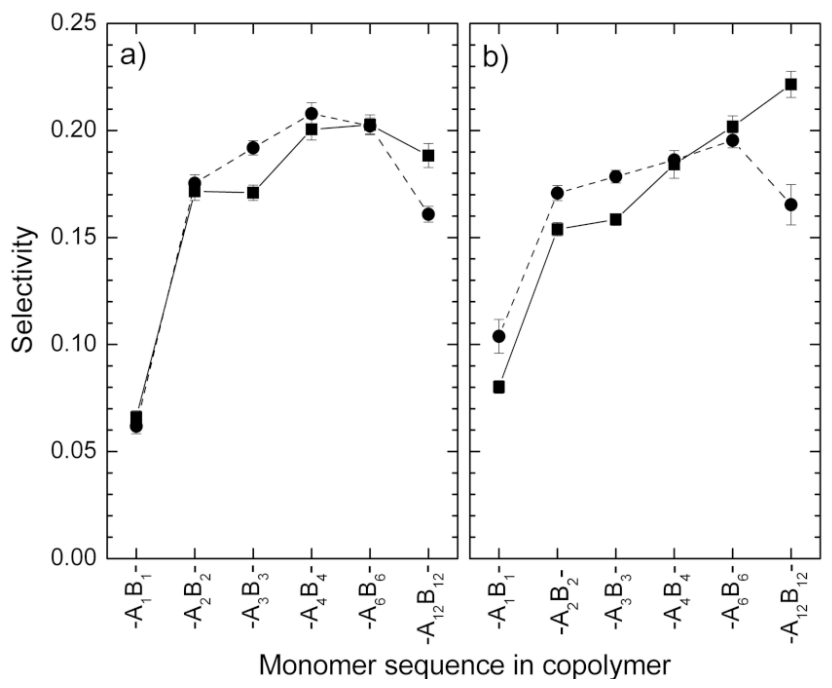

FIG. 3. The selectivity of the surfaces designed for (a) $\left[A_{4} B_{4}\right]$ copolymer with $0 \%$ (solid line) and $10 \%$ (dashed line) neutral sites and (b) $\left[A_{6} B_{6}\right]$ copolymer at $0 \%$ (solid line) and $30 \%$ (dashed line) neutral sites, using set III interactions.

In order to test the efficiency of our design method we compare the selectivity of the designed surfaces that displayed the best pattern recognition to the selectivity of the checkerboard surface having pattern sizes that are commensurate with the size of the sequences in the copolymers (Fig. 4). The $1 \times 1$ checkerboard surface has a selectivity equal to 1.00 for $\left[A_{1} B_{1}\right]$ copolymers while the designed surface has a selectivity of 0.50 for $\left[A_{1} B_{1}\right]$ copolymers. Since the $1 \times 1$ checkerboard surface (order parameter $-1.00)$ is a perfectly alternating surface, the only way that our design algorithm could generate such a pattern is if in the design step the $\left[A_{1} B_{1}\right]$ copolymers get adsorbed in perfectly flat uncoiled conformations and lie next to each other in a parallel alignment; this does not happen due to low probability associated with the generation of such conformations in our simulations. A similar problem leads to the result that the $2 \times 2$ checkerboard surface has higher selectivity $(0.64)$ for $\left[A_{2} B_{2}\right]$ copolymers than the designed surface (0.28). In contrast, for $\left[A_{3} B_{3}\right]$ [Fig. 4(c)], $\left[A_{4} B_{4}\right]$ [Fig. 4(d)], and $\left[A_{6} B_{6}\right]$ [Fig. 4(e)] copolymers, our designed surfaces exhibit better pattern recognition than the corresponding checker board surfaces. This is because the checkerboard surfaces have a patchiness higher than is required for the recognition of the corresponding commensurate monomer sequences. Our design simulation generates surfaces with patchiness closer to the value required for the recognition of corresponding sequences. It is important to point out that our design method yields an ensemble of optimal surfaces rather than the single best surface. The latter, if it exists, would likely be a member of this ensemble. Our design method should prove useful in exploring the relationship between the spatial distribution of chemical heterogeneities in biopolymers and the adsorbing surface and thus contribute to current understanding of transmembrane signaling, pathogen-host interactions, etc.

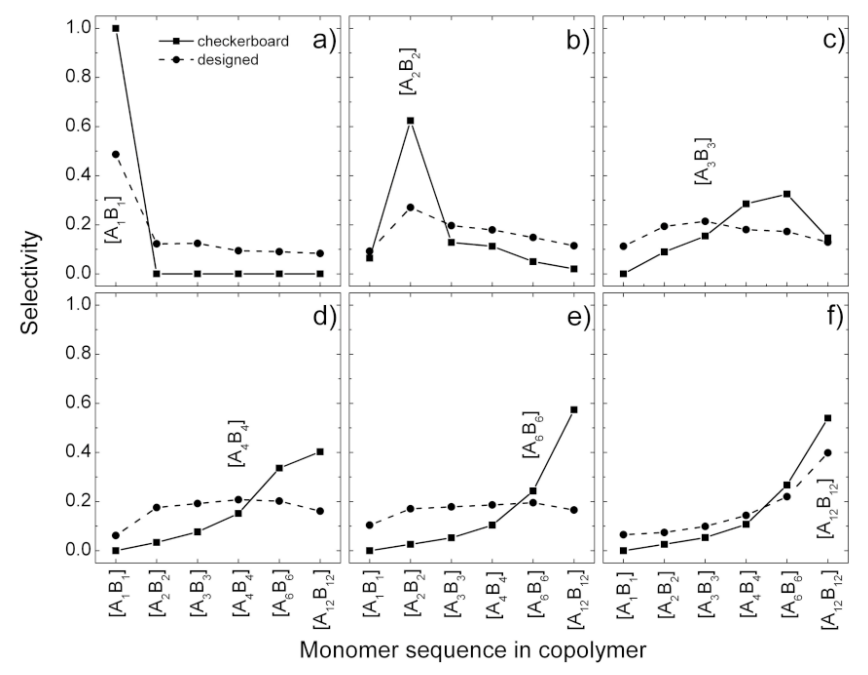

FIG. 4. Selectivity of checkerboard (squares solid line) and designed surfaces (circles dashed line) for the different copolymers with set III interactions except as noted (a) $1 \times 1$ vs surface designed for $\left[A_{1} B_{1}\right]$ with $0 \%$ neutral sites, (b) $2 \times 2$ vs surface designed for $\left[A_{2} B_{2}\right]$ with $10 \%$ neutral sites, (c) $3 \times 3$ vs surface designed for $\left[A_{3} B_{3}\right]$ with $0 \%$ neutral sites, (d) $4 \times 4$ vs surface designed for $\left[A_{4} B_{4}\right]$ with $10 \%$ neutral sites, (e) $6 \times 6$ vs surface designed for $\left[A_{6} B_{6}\right]$ with $30 \%$ neutral sites, and (f) $12 \times 12$ vs surface designed for $\left[A_{12} B_{12}\right]$ with $0 \%$ neutral sites using set I interactions.

The support of the Office of Energy Research, U.S. Department of Energy under Contract No. DE-FG0591ER14181 (C. K.H.), and the National Science Foundation under Grant No. DMR-0353102 (J.G.) is gratefully acknowledged.

*Electronic address: hall@eos.ncsu.edu

[1] S. Walheim, E. Schaffer, J. Mlynek, and U. Steiner, Science 283, 520 (1999).

[2] R.J. Todd, R.D. Johnson, and F.H. Arnold, J. Chromatogr., A 662, 13 (1994).

[3] R. F. Service, Science 270, 230 (1995).

[4] D. Bratko, A. K. Chakraborty, and E. I. Shakhnovich, Comput. Theor. Polym. Sci. 8, 113 (1998).

[5] S. Srebnik, J. Chem. Phys. 114, 9179 (2001).

[6] A. K. Chakraborty and D. Bratko, J. Chem. Phys. 108, 1676 (1998).

[7] A. J. Golumbfskie, V.S. Pande, and A. K. Chakraborty, Proc. Natl. Acad. Sci. U.S.A. 96, 11707 (1999).

[8] K. Haupt, Nat. Biotechnol. 20, 884 (2002).

[9] N. A. Peppas and Y. Huang, Pharmaceutical research 19, 578 (2002).

[10] J. Genzer, Adv. Colloid Interface Sci. 94, 105 (2001).

[11] J. Genzer, Phys. Rev. E 63, 022601 (2001).

[12] C. Seok, K. F. Freed, and I. Szleifer, J. Chem. Phys. 112, 6443 (2000).

[13] M. Muthukumar, J. Chem. Phys. 103, 4723 (1995).

[14] M. Muthukumar, Proc. Natl. Acad. Sci. U.S.A. 96, 11690 (1999).

[15] S. Srebnik, J. Chem. Phys. 112, 9655 (2000). 